\title{
Double-Walled Airbag Breast Immobilization Device
}

National Cancer Institute

\section{Source}

National Cancer Institute. Double-Walled Airbag Breast Immobilization Device. NCI

Thesaurus. Code C126757.

A device that utilizes an inflatable double-walled airbag to establish and maintain the breast in a fixed and well-defined position during radiotherapy. 